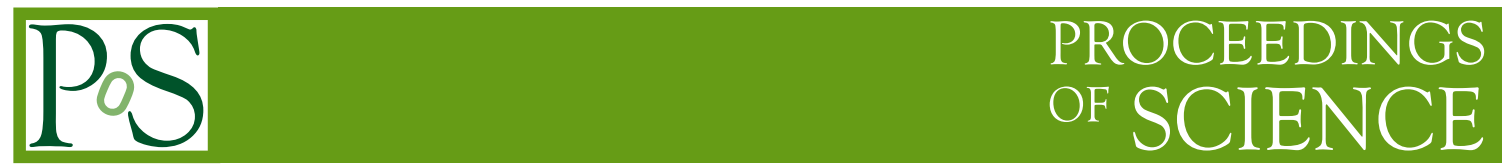

\title{
The cosmological constant and quantum vacuum
}

\author{
Alain Blanchard* \\ Institut de recherche en astrophysique et planétologie \\ E-mail: alain.blanchardeirap.omp.eu
}

\begin{abstract}
The acceleration of the expansion can now be regarded as established beyond reasonable doubt. However, the physical origin of this acceleration, the so-called dark energy, remains a puzzling problem in fundamental physics. The current explanations, quintessence and modified gravity, are based on a fundamental revision of known physics.

Here I will present a revival of an old proposition : that the origin of cosmic acceleration is due to the gravitational active vacuum.
\end{abstract}

Frontiers of Fundamental Physics 14 - FFP14,

15-18 July 2014

Aix Marseille University (AMU) Saint-Charles Campus, Marseille

\footnotetext{
*Speaker.
} 